\title{
L-Galactosa deshidrogenasa y L-Gulono-1,4-lactona deshidrogenasa influyen en la biosíntesis de vitamina C en Myrciaria dubia (Kunth) McVaugh "camu-camu"
}

\section{L-Galactose dehydrogenase and L-Gulone-1,4-lactone dehydrogenase influence in biosynthesis of vitamin C in Myrciaria dubia (Kunth) McVaugh "camu-camu"}

\author{
Juan C. Castro ${ }^{1}$, Angel Araujo ${ }^{1}$, Freddy Gutiérrez ${ }^{1}$, Sixto A. Imán²
}

${ }^{1}$ Laboratorio de Biotecnología. Centro de Investigaciones de Recursos Naturales de la Amazonía (CIRNA), Universidad Nacional de la Amazonia Peruana (UNAP). Pasaje Los Paujiles S/N. AAHH Nuevo San Lorenzo.

Distrito de San Juan Bautista-Iquitos

${ }^{2}$ Instituto Nacional de Innovación Agraria (INIA). San Roque. Iquitos-Perú.

\section{Resumen}

La vitamina $C$ es un nutriente esencial para el hombre y una de sus fuentes naturales son los frutos de Myrciaria dubia. Pero esta planta muestra amplia variación en contenido de vitamina C. Para ayudar a esclarecer las bases bioquímicas de esta variación, nos planteamos como objetivos determinar si las enzimas L-Galactosa deshidrogenasa (L-GalDH) y L-Gulono-1,4-lactona deshidrogenasa (L-GuLDH) están presentes en las hojas y frutos (pulpa y cáscara) del camu-camu y comparar la actividad catalítica de ambas enzimas en la pulpa de frutos cosechados de plantas que producen alto y bajo contenido de vitamina $\mathrm{C}$. Las hojas y frutos fueron obtenidos de seis plantas (tres que producen bajo y tres que producen alto contenido de vitamina $\mathrm{C}$ ) de la colección de germoplasma del INIA. La vitamina C fue extraída y cuantificada mediante HPLC. Posteriormente, de ambos grupos de plantas, se purificó parcialmente las enzimas y midió por triplicado su actividad catalítica mediante espectrofotometría. Tanto L-GalDH como L-GuLDH fueron detectadas en las hojas, pulpa y cáscara. Ambas enzimas mostraron diferencias en sus actividades catalíticas entre los tejidos analizados $(p<0,05)$. Al comparar la actividad de estas enzimas entre plantas que producen frutos con alto $(2258 \pm 217 \mathrm{mg}$ Vit. C/100g pulpa) y bajo (1570 $\pm 46 \mathrm{mg}$ Vit. C/100g pulpa) contenido de vitamina $C$ se encontró diferencias significativas $(p<0,05)$ entre ambos grupos. Siendo mayor la actividad catalítica de L-GalDH y L-GuLDH en frutos con alto contenido de vitamina $\mathrm{C}$ y mostraron menor actividad catalítica en los frutos con bajo contenido de esta vitamina. Adicionalmente, ensayos cinéticos realizados con la L-GuLDH del camu-camu mostraron que esta enzima tiene una alta afinidad $\left(K_{\mathrm{m}}=2,37 \mu \mathrm{M}\right.$ y $\left.V_{\max }=9,23 \mu \mathrm{mol} \cdot \mathrm{mg} \mathrm{prot}^{-1} \cdot \mathrm{min}^{-1}\right)$ por su sustrato L-Gulono-1,4-lactona. En conclusión, las enzimas L-Galactosa deshidrogenasa y L-Gulono-1,4-lactono deshidrogenasa están presentes en las hojas y frutos del camu-camu, lo que nos indica que las dos vías biosintéticas de vitamina $\mathrm{C}$ propuestas para las plantas (vía de Smirnoff-Wheeler y vía de Wolucka), determinan la producción de vitamina C en M. dubia. Asimismo, las evidencias experimentales sugieren que la acumulación de vitamina $C$ en la pulpa de los frutos del camu-camu depende por lo menos de dos procesos: biosíntesis in situ y transporte desde otros tejidos con capacidad biosintética. Además, se evidencia que la actividad catalítica de L-GalDH y L-GulDH influyen en el contenido de vitamina $C$ de la pulpa, de tal modo que una mayor actividad catalítica de ambas enzimas está asociada con más contenido de vitamina $\mathrm{C}$ en este tejido. 
Palabras clave: L-galactosa deshidrogenasa, L-gulono-1,4-lactona deshidrogenasa, biosíntesis de vitamina C, Myrciaria dubia

\section{Abstract}

Vitamin $\mathrm{C}$ is an essential nutrient for humans and one their natural sources are fruits of Myrciaria dubia. But this plant shows wide variation in vitamin $\mathrm{C}$. To help clarify the biochemical basis of this variation, we plan aims to determine whether the enzymes L-Galactose dehydrogenase (L-GalDH) and L-Gulone-1,4-Lactone dehydrogenase (L-GuLDH) are presents in leaves and fruits (pulp and peel) of camu-camu and compare catalytic activity of both enzymes in pulp of fruits harvested from plants that produce high and low content of vitamin $\mathrm{C}$. Leaves and fruits were obtained from six plants (three producing low and three that produce high content of vitamin $\mathrm{C}$ ) in the germplasm collection of INIA. Vitamin C was extracted and quantified by HPLC. Subsequently, from both groups of plants, was partially purified enzymes and measured by triplicate their catalytic activity by means of spectrophotometry. Both L-GalDH and L-GuLDH were detected in leaves, pulp and peel. Both enzymes showed differences in their catalytic activities among the tissues analyzed $(p<0.05)$. By comparing the activity of

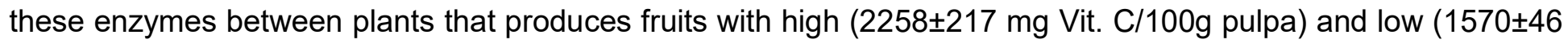
mg Vit. C/100g pulpa) content of vitamin $C$ significant differences were found $(p<0.05)$ between groups. Being higher the catalytic activity of L-GalDH and L-GuLDH in fruits with high content in vitamin C and showed lower catalytic activity in fruits with low content in this vitamin. In addition, kinetic assays performed with the L-GuLDH of camu-camu showed that this enzyme has a high affinity $\left(K_{\mathrm{m}}=2.37 \mu \mathrm{M}\right.$ y $\left.V_{\max }=9.23 \mu \mathrm{mol} \cdot \mathrm{mg} \mathrm{prot}^{-1} \cdot \mathrm{min}^{-1}\right)$ for its substrate L-Gulone-1,4-lactone. In conclusion, the enzymes L-galactose dehydrogenase and L-gulone-1,4-lactone dehydrogenase are present in leaves and fruits of camu-camu, which indicates that the two biosynthetic pathways of vitamin C proposals for plants (pathway of Smirnoff-Wheeler and pathway of Wolucka) determine production of vitamin $C$ in M. dubia. Furthermore, experimental evidences suggest that the accumulation of vitamin $\mathrm{C}$ in the pulp of the fruits of camu-camu depends at least of two processes: in situ biosynthesis and transport from other tissues with biosynthetic capacity. Furthermore, it is evident that catalytic activity of L-GalDH and L-GulDH influence in vitamin $\mathrm{C}$ content of pulp, so that a higher catalytic activity of both enzymes is associated with more vitamin $\mathrm{C}$ content in this tissue.

Keywords: L-galactose dehydrogenase, L-gulone-1,4-lactone dehydrogenase, biosynthesis of vitamin C, Myrciaria dubia

\section{Introducción}

La vitamina $\mathrm{C}$ es un compuesto esencial para el hombre, debido a sus roles esenciales como antioxidante y cofactor de varias enzimas [1]. Por tanto, su aumento en la dieta proporciona beneficios significativos en la salud [2], [3].

Una fuente importante de vitamina C para el consumo humano son los frutos de Myrciaria dubia "camu-camu". Este frutal nativo de la región amazónica se distingue porque sus frutos tienen elevado contenido de vitamina C [4]. Sin embargo, hay reportes que indican una amplia variación en el contenido de esta vitamina en los frutos obtenidos de diferentes plantas de rodales naturales [5] y del banco de germoplasma del INIA [6]. Adicionalmente, se ha demostrado que la cáscara de los frutos tiene mayor concentración de vitamina $C$ que la pulpa. Además, las hojas del camu-camu presentan en promedio $433 \mathrm{mg}$ vitamina $\mathrm{C} / 100 \mathrm{~g}$ de hoja [7].

A pesar de la importancia del camu-camu en la producción de vitamina $\mathrm{C}$, no existen reportes de estudios bioquímicos y/o moleculares que permitan explicar el proceso de biosíntesis y factores que influyen en la producción de esta vitamina. Pero, investigaciones realizadas por Smirnoff-Wheeler [8] y Wolucka [9] indican la existencia de dos vías 
metabólicas responsables de la biosíntesis de vitamina $\mathrm{C}$ en plantas.

En ese sentido, los objetivos de esta investigación fueron determinar si las enzimas L-Galactosa deshidrogenasa (L-GalDH) y L-Gulono-1,4-lactona deshidrogenasa (L-GuLDH) están presentes en las hojas y frutos (pulpa y cáscara) del camu-camu y comparar la actividad catalítica de ambas enzimas en la pulpa de frutos cosechados de plantas que producen alto y bajo contenido de vitamina $\mathrm{C}$.

\section{Metodología experimental}

\section{Colecta de material botánico}

Las hojas y frutos pintones (pulpa y cáscara) fueron obtenidos de seis plantas, que previamente han sido determinadas por [6] que tienen frutos con bajo contenido de vitamina $\mathrm{C}$ y tres con alto contenido de vitamina C, de la Colección Nacional de Germoplasma de M. dubia del Instituto Nacional de Innovación Agraria (INIA), ubicado en el Km $25 \frac{1}{2}$ de la carretera lquitos-Nauta $\left(03^{\circ} 57^{\prime} 23^{\prime \prime}\right.$ LS y $73^{\circ} 24^{\prime}$ $47^{\prime \prime}$ LO). La colecta se realizó entre las 8-10 horas del mes de abril del 2011. Las muestras colectadas fueron puestas en bolsas plásticas rotuladas y transportadas en refrigeración (en termos con hielo) al laboratorio de Biotecnología del CIRNA, donde fueron almacenados a $-20^{\circ} \mathrm{C}$ hasta su procesamiento.

\section{Determinación de vitamina C}

Se realizó de acuerdo a Ledezma [10]. Consistió en triturar, en un mortero, $250 \mathrm{mg}$ de la muestra botánica (hoja, pulpa ó cáscara) al que se agregó $0,95 \mathrm{ml}$ de solución de extracción $\left(\mathrm{HPO}_{3} 3 \%\right.$, ácido acético $8 \%$ y EDTA 0,36\%). El homogenizado se centrifugó en microtubos de $1,5 \mathrm{ml}$ a $15000 \mathrm{rpm}$ por $10 \mathrm{~min}$, el sobrenadante obtenido se diluyó diez veces (excepto el obtenido de las hojas), se filtró a través de un filtro para jeringa de 0,45 $\mu \mathrm{m}$. Diez microlitros de las muestras filtradas fueron analizadas en un HPLC (Elite La Chrome, modelo HP 1100), empleando una columna RP-18 atemperada a $56{ }^{\circ} \mathrm{C}$. El flujo de la fase móvil
$\left(\mathrm{KH}_{2} \mathrm{PO}_{4} 100 \mathrm{mM} \mathrm{pH} 2,5\right)$ fue $0,7 \mathrm{ml} / \mathrm{min}$. La vitamina C fue detectada a $245 \mathrm{~nm}$ y se cuantificó en base a la curva estándar obtenida.

\section{Purificación parcial de enzimas}

Se realizó en base a [11], [12] y [13]. Para ello, $1,5 \mathrm{~g}$ de la muestra (hojas, pulpa ó cáscara) fue triturada, en un mortero estéril, añadiendo progresivamente 5 $\mathrm{ml}$ de buffer de extracción (Tris-HCl $100 \mathrm{mM} \mathrm{pH:} \mathrm{7,5,}$ $\mathrm{MgCl}_{2} 1 \mathrm{mM}$, EDTA $2 \mathrm{mM}$, DTT $2 \mathrm{mM}$, ácido aminocaproico $1 \mathrm{mM}$, benzamedina hidrocloruro 1 $\mathrm{mM}$, PMSF $1 \mathrm{mM}$, PVP $3,0 \%$ y glicerol $20 \%$ ). Luego, el triturado obtenido fue filtrado a través de tres capas de miracloth. El producto obtenido fue centrifugado a $21300 \mathrm{~g}$ por $15 \mathrm{~min}$ a $4{ }^{\circ} \mathrm{C}$ y el sobrenadante fue transferido a una membrana (MWCO 6,000-8,000 FisherBrand) para su diálisis con $500 \mathrm{ml}$ de tampón (Tris-HCl $50 \mathrm{mM} \mathrm{pH} \mathrm{7,5} \mathrm{y} 1$ $\mathrm{mM}$ DTT) por $12 \mathrm{~h}$. El producto de la diálisis fue puesto en microtubos de $1,5 \mathrm{ml}$ para su centrifugación a $21300 \mathrm{~g}$ por $5 \mathrm{~min}$ a $4{ }^{\circ} \mathrm{C}$. El sobrenadante obtenido fue almacenado en alícuotas de $100 \mu \mathrm{l} \mathrm{a}-20^{\circ} \mathrm{C}$.

La cuantificación de las proteínas totales se realizó según [14].

\section{Medición de la actividad enzimática L-Galactosa deshidrogenasa (L-GalDH)}

Se determinó según [11] y [15]. Los ensayos estándares contenían Tris $\mathrm{HCl} 100 \mathrm{mM} \mathrm{pH} \mathrm{8,0,} \mathrm{NAD}$ $1 \mathrm{mM}$ y L-Galactosa $1 \mathrm{mM}$. La reacción se inició por la adición de $100 \mu \mathrm{l}$ del extracto enzimático, registrándose la absorbancia a $340 \mathrm{~nm}$ (en un espectrofotómetro UV/Vis Genesys 6) cada $30 \mathrm{seg}$ por 6 a 10 min a $25^{\circ} \mathrm{C}$. El control negativo de los ensayos contenía $100 \mu \mathrm{l}$ del extracto enzimático inactivo (tratado a $95{ }^{\circ} \mathrm{C}$ por $10 \mathrm{~min}$ ) y los demás componentes de la reacción. Los valores de la velocidad inicial fueron calculados usando un coeficiente de extinción molar de $6,2 \mathrm{mM}^{-1} \cdot \mathrm{min}^{-1}$.

\section{L-Gulono-1,4-lactona deshidrogenasa (L-GuLDH)}

Se realizó según [16]. Los ensayos estándares contenían tampón fosfato $10 \mathrm{mM} \mathrm{pH} \mathrm{7,8,} \mathrm{Triton} \mathrm{X-}$ 100 0,03 \% Cianuro de potasio $0,1 \mathrm{mM}$, citocromo c 
$1,0 \mathrm{mg} / \mathrm{ml}$, L-Gulono-1,4-lactona $0,5 \mathrm{mM}$. La reacción se inició por la adición de $100 \mu \mathrm{l}$ del extracto enzimático, registrándose la absorbancia a $540 \mathrm{~nm}$ cada $30 \mathrm{seg}$ por 6 a $10 \mathrm{~min}$ a $25^{\circ} \mathrm{C}$. El control negativo de los ensayos contenía $100 \mu \mathrm{l}$ del extracto enzimático inactivo (tratado a $95^{\circ} \mathrm{C}$ por 10 min) y los demás componentes de la reacción. Los valores de velocidad inicial fueron calculados usando la diferencia del coeficiente de extinción molar $(\Delta \varepsilon)$ de $21 \mathrm{mM}^{-1} \cdot \mathrm{cm}^{-1}$ para el citocromo $\mathrm{c}$ reducido menos el oxidado.

Con las medidas de velocidad inicial de la enzima en un intervalo de 0,0001 a $10 \mathrm{mM}$ de L-Gulono-1,4lactona, se obtuvo la ecuación de Lineweaver-Burk: $\frac{1}{V_{0}}=0,2573 \frac{1}{[S]}+0,1084$ con un $R^{2}=0,9997$. En base a esta ecuación se estimaron los parámetros cinéticos $\left(K_{\mathrm{m}}\right.$ y $\left.V_{\max }\right)$ de la enzima.

\section{Análisis de datos}

Los datos obtenidos fueron almacenados en una hoja de Microsoft Excel 2010. Los análisis estadísticos (promedio, error estándar de la media y prueba t-Student) se realizaron con el programa IBM SPSS $\vee 19$.

\section{Resultados Y Discusión}

El contenido de vitamina $\mathrm{C}$ en los tres tejidos analizados fue variable. Siendo menor en las hojas (380 $\pm 50 \mathrm{mg}$ Vit. C/100 g hoja), mayor en la pulpa (1616 $\pm 454 \mathrm{mg}$ Vit. C/100 g pulpa) y el valor más alto se registró en la cáscara $(1987 \pm 615 \mathrm{mg}$ Vit. C/100 g cáscara).

La variación en el contenido de vitamina $\mathrm{C}$ puede deberse, en parte, a diferencias en la actividad catalítica de las enzimas L-GalDH y L-GuLDH. Ambas enzimas fueron detectadas en los tres tejidos analizados del camu-camu (Fig. 1). Sin embargo, sus actividades catalíticas mostraron diferencias significativas (prueba t-Student, $p<0,05$ ), de tal modo que L-GalDH (Fig. 1A) fue más activa en cáscara y hojas, pero manifestó menor actividad catalítica en la pulpa. Similarmente, L-GuLDH (Fig.
1B) también exhibió actividad diferencial entre los tejidos (prueba t-Student, $p<0,05$ ), siendo más alta en pulpa y cáscara, pero mostró baja actividad en hojas.
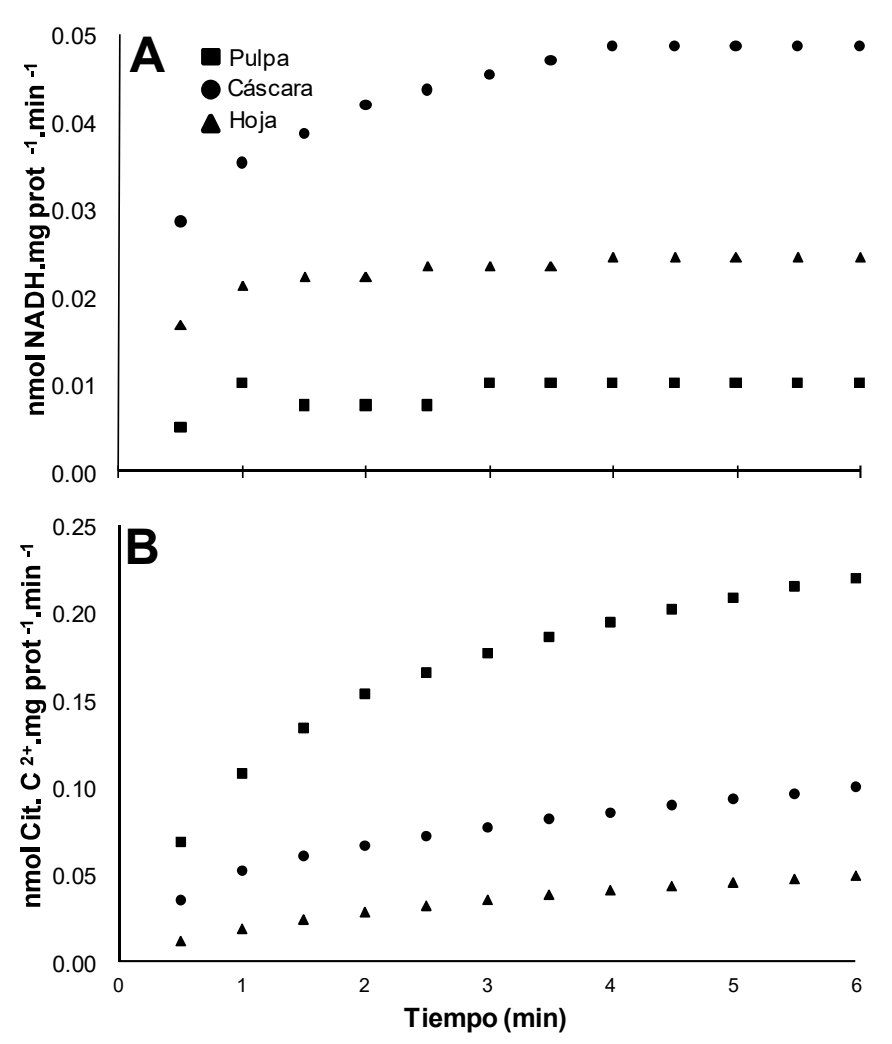

Fig 1. Actividad catalítica de L-GalDH (A) y L-GuLDH (B) en hoja, pulpa y cáscara de $M$. dubia "camu-camu"

Estos resultados nos sugieren que en el camu-camu operan dos vías de biosíntesis de vitamina C: la propuesta por Smirnoff-Wheeler [8], representada en este estudio por L-GalDH y la ruta alternativa planteada por Wolucka [9], representada por LGuLDH. Además, los hallazgos nos sugieren que los frutos (pulpa y cáscara) tienen capacidad biosintética de vitamina $\mathrm{C}$, en consecuencia, es posible que el contenido de esta vitamina en la pulpa de frutos del camu-camu dependa de su producción in situ y transporte desde las hojas y cáscara. 
Otros estudios también han detectado la actividad de las enzimas mencionadas en varias especies. Así, la L-GalDH está presente en hojas de Arabidopsis thaliana [11], Spinacia oleracea [17] y Actinidia deliciosa [14]. Asimismo, Hancok [12] encuentra esta enzima en el floema y tejidos vasculares de Apium graveolens, Cucumis sativus y cinco especies del género Cucurbita. En contraste, los reportes de LGuLDH son más escasos. Entre estos, Wolucka [9] encuentra actividad de esta enzima en la fracción citosólica y mitocondrial de tubérculos de Solanum tuberosum. Asimismo, se reporta la presencia de LGuLDH en Mycobacterium tuberculosis [18].

Las evidencias experimentales sugieren que los niveles de actividad catalítica de las enzimas LGalDH y L-GuLDH influyen en el contenido de vitamina $C$ de la pulpa. Al comparar la actividad de ambas enzimas entre plantas que producen frutos con alto y bajo contenido de vitamina $C$, se encontró diferencias significativas (prueba t-Student, $p<0,05$ ) entre ambos grupos. Siendo el patrón común una mayor actividad catalítica de L-GalDH y L-GuLDH en frutos con alto contenido de vitamina $C$ y menor actividad catalítica en los frutos con bajo contenido de esta vitamina (Fig. 2).

En principio, las diferencias en la actividad catalítica se pueden atribuir a varios factores que afectan la cantidad de estas enzimas presentes en los tejidos analizados. Entre estos, el nivel de expresión de los genes que codifican estas enzimas podría ser mayor en los frutos con alto contenido de vitamina $C$ en contraste a los frutos que presentan bajo contenido. Estas diferencias en el nivel de expresión estarían influyendo en la cantidad de enzima sintetizada, es decir, se espera que los tejidos con mayor número de ARNm codantes de L-GalDH y L-GuLDH, presentan mayor traducción de estas enzimas y por ende tendrían más moléculas enzimáticas que incrementarían su capacidad para sintetizar vitamina C.

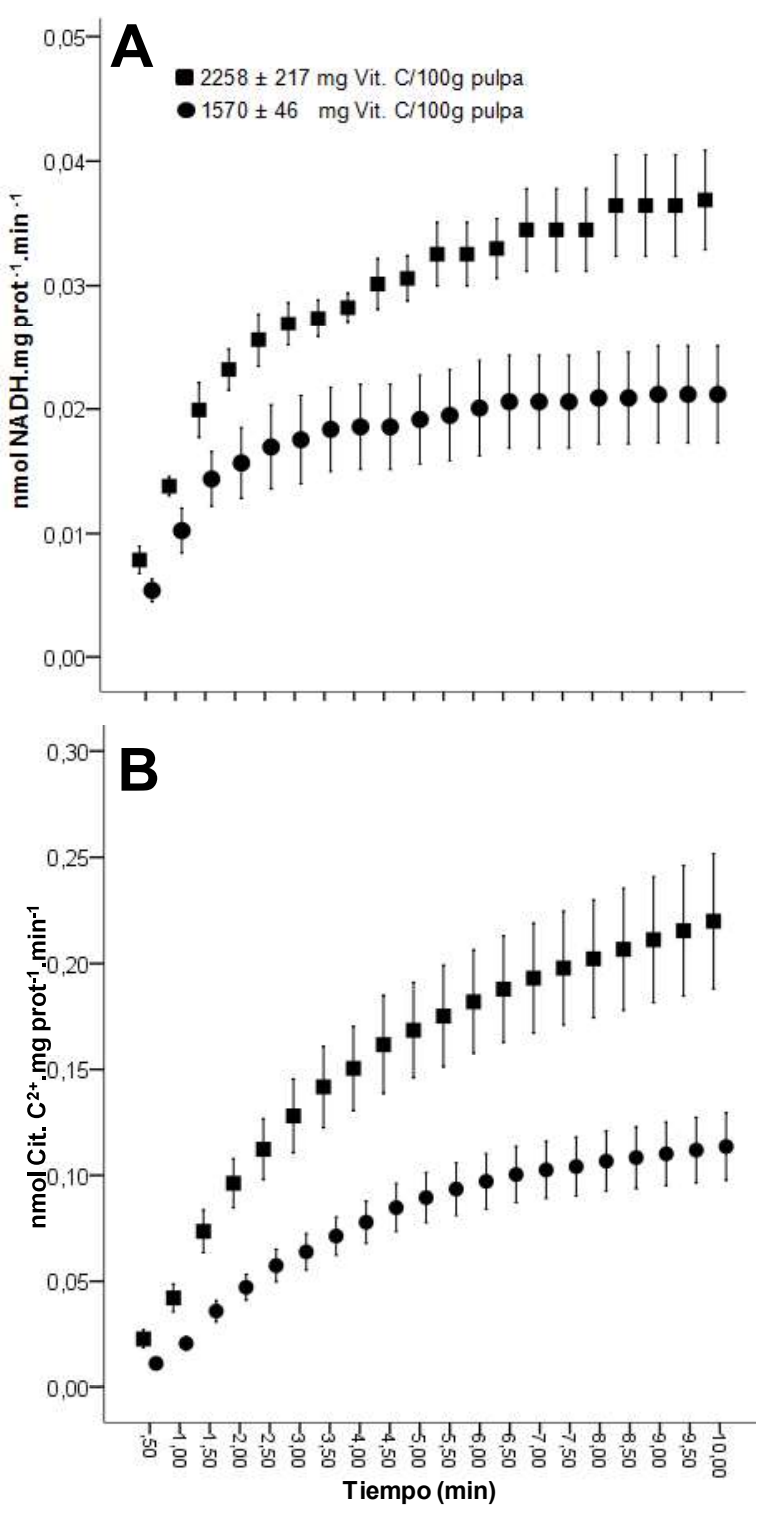

Fig 2. Comparación de la actividad catalítica de L-GaIDH (A) y L-GuLDH (B) en pulpa de frutos de $M$. dubia "camu-camu" que presentan alto y bajo contenido de vitamina $\mathrm{C}$

Por otra parte, la enzima L-GuLDH del camu camu presentó parámetros cinéticos excepcionales. Por ejemplo, mostró una alta afinidad por su sustrato (LGulono-1,4-lactona), la que se manifestó por su baja constante de Michaelis $\left(K_{\mathrm{m}}=2,37 \mu \mathrm{M}\right)$ y $V_{\max }=9,23$ $\mu \mathrm{mol}$.mg $\operatorname{prot}^{-1}$. $\mathrm{min}^{-1}$ (Fig.3). 


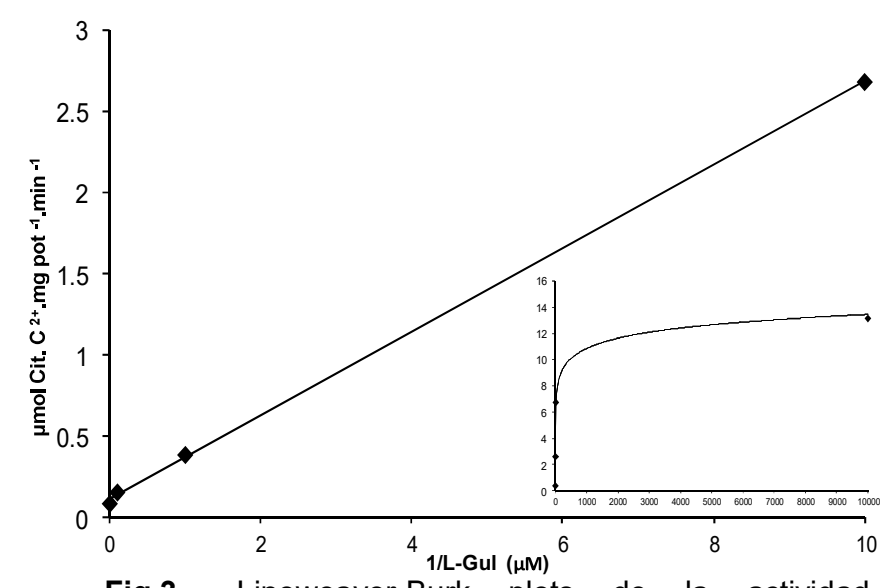

Fig 3. Lineweaver-Burk plots de la actividad catalíticade L-GuLDH en función a la concentración de L-Gulono-1,4-lactona

Estas evidencias nos sugieren que la enzima detectada en los tres tejidos del camu- camu es la LGulono-1,4-lactona deshidrogenasa y no la enzima L-Galactono-1,4-lactona deshidrogenasa. Las diferencias entre estas enzimas se pueden juzgar al comparar la afinidad que tienen por sus sustratos. De acuerdo a lo mencionado, Leferink et al. [19] muestra que la enzima L-Galactono-1,4-lactona deshidrogenasa de Arabidopsis thaliana tiene un $\mathrm{Km}$ de $0,17 \pm 0,01 \mathrm{mM}$ para L-Galactono-1,4-lactona, mientras que para L-Gulono-1,4-lactona, el cual no es su sustrato, presenta un $K_{\mathrm{m}} 77$ veces mayor $\left(K_{\mathrm{m}}=\right.$ $13,1 \pm 2,8 \mathrm{mM})$. Pero, para confirmar la identidad de la L-GuLDH presente en los tejidos del camu-camu es necesario purificar la enzima y comparar su $\mathrm{K}_{\mathrm{m}} \mathrm{y}$ $\mathrm{V}_{\text {max }}$ para ambos sustratos.

\section{CONCLUSIONES}

Las enzimas L-Galactosa deshidrogenasa y LGulono-1,4-lactono deshidrogenasa están presentes en las hojas y frutos del camu-camu, lo que nos indica que las dos vías biosintéticas de vitamina $\mathrm{C}$ propuestas para las plantas (vía de SmirnoffWheeler y vía de Wolucka), determinan la producción de vitamina C en M. dubia. Asimismo, las evidencias experimentales sugieren que la acumulación de vitamina $\mathrm{C}$ en la pulpa de los frutos del camu-camu depende por lo menos de dos procesos: biosíntesis in situ y transporte desde otros tejidos con capacidad biosintética. Además, se evidencia que la actividad catalítica de L-GalDH y LGulDH influyen en el contenido de vitamina $C$ de la pulpa, de tal modo que una mayor actividad catalítica de ambas enzimas está asociada con más contenido de vitamina $\mathrm{C}$ en este tejido.

\section{Agradecimientos}

Al Consejo Nacional de Ciencia, Tecnología e Innovación Tecnológica (CONCYTEC) y Universidad Nacional de la Amazonía Peruana (UNAP) por el apoyo financiero. También agradecemos a los estudiantes Alex Tapullima Pacaya y Cinthya Acuña Amaral por la contribución experimental.

\section{Referencias}

[1] C. Cronjé. Thesis presented in partial fulfillment of the requirements for the degree Master of Sciencie. University of Stellenbosch. 2010.

[2] A. C. Carr, B. Frei. American Journal of Clinical Nutrition 69 (1999) 1086-1107.

[3] M. Levine, S. C. Rumsey, R. Daruwala, J. B. Park, Y. H. Wang. Journal of the American Medical Association 281 (1999) 1415-1423.

[4] C. S. Iman. Manual No 01-00. PRONARGEBINIA. 2000.

[5] M. Pinedo, H. Inga, S. Pinedo, y C. Linares. Programa de Ecosistemas Terrestres. Informe de colección de germoplasma. 2002.

[6] J. Castro, M. Cobos, R. Ramírez, A. Egoávil, F. Gutierrez, F. Torres, S. Imán, R. Rojas, M. Siguas, A. Córdova, P. Adriánzén, J.Marapara. Encuentro científico del Consejo Nacional de Ciencia y Tecnología. 2011.

[7] A. M. Córdova, R. Ramírez, M. Cobos, S. A. Imán, J. C. Castro. UNAMAZ. 2010

[8] G. L Wheeler, M. A. Jones, N. Smirnoff. 
Nature 393 (1998) 365-369.

[9] B. A Wolucka, M. Van Montagu. J Biol Chem. 278 (2003) 47483-47490.

[10] M. LEDEZMA. Tecnología en Marcha. 2004.

[11] S. Gatzek, G. L. Wheeler, N Smirnoff. The Plant journal 30 (2002) 541-553.

[12] R. D. Hancock, D. Mcrae, S. Haupt, R. Viola. Plant Biology. (2003) 1-13.

[13] P. L. Conklin, S. Gatzek,G. L. Wheeler, J. Dowdle, M. Raymond, S. Rolinski, M. Isupov, J. A. Littlechild, N. Smirnoff. The Journal Biological Chemistry. 281(2006)15662-15670.

[14] S. Roe. Oxford University Press. 2006.

[15] W. A. Laing, N. Frearson, S. Bulley, E. Macrae. Functional plant biology. 31(2004) 1015-1025.
[16] K. Oba, S. Ishikawa, M. Nishikawa, H. Mizuno, T. Yamamoto.Biochem. J. 117(1995) 120-124.

[17] T. Mieda, Y. Yabuta, M. Rapolu, T. Motoki, T. Takeda, K. Yoshimura, T. Ishikawa and S. Shigeoka. Plant Cell Physiol. 45(2004) 12711279.

[18] B. A. Wolucka, D. Communi. FEBS J. 273(2006) 4435-45.

[19] N. G. H. Leferink, W. A. M. van den Berg, W. J. H. van Berkel. FEBS J. 275(2008) 713-726.

juanccgomez@yahoo.es 